\title{
BMJ Open Prevalence of depression symptoms and its influencing factors among pregnant women in late pregnancy in urban areas of Hengyang City, Hunan Province, China: a cross-sectional study
}

To cite: Yu Y, Zhu X, Xu H, et al. Prevalence of depression symptoms and its influencing factors among pregnant women in late pregnancy in urban areas of Hengyang City, Hunan Province, China: a crosssectional study. BMJ Open 2020;10:e038511. doi:10.1136/ bmjopen-2020-038511

- Prepublication history for this paper is available online. To view these files, please visit the journal online (http://dx.doi. org/10.1136/bmjopen-2020038511).

$\mathrm{YY}$ and $\mathrm{XZ}$ are joint first authors.

Received 16 March 2020

Revised 02 June 2020

Accepted 29 July 2020

Check for updates

(c) Author(s) (or their employer(s)) 2020. Re-use permitted under CC BY-NC. No commercial re-use. See rights and permissions. Published by BMJ.

Department of Social Medicine and Health Management, Centra South University Xiangya School of Public Health, Changsha, Hunan, China

Correspondence to

Dr Huilan Xu;

XHL202020@163.com

\section{ABSTRACT}

Objectives To evaluate the prevalence of depressive symptoms and its influencing factors in late pregnancy. Design Cross-sectional study.

Setting Fourteen community in urban areas of Hengyang City.

Participants The study conducted from July to October 2019 , and surveyed 813 women in late pregnancy who lived in urban areas of Hengyang for more than 6 months, signed an informed consent and were without cognitive disorders, severe mental illnesses or other serious diseases.

Measures Perinatal depression symptoms were evaluated using the Patient Health Questionnaire-9, and perinatal anxiety symptoms were evaluated using the Generalised Anxiety Disorder-7 Scale. Sociodemographic variables, obstetric characteristics, lifestyle behaviours, family factors, social support, sleep quality and self-efficacy were obtained through structured questionnaires.

Results The prevalence of depression symptoms among pregnant women in late pregnancy was $9.2 \%(95 \% \mathrm{Cl}$ $7.2 \%-11.2 \%)$. Protective factor: age between 25 and 29 years $(0 \mathrm{R}=0.398 ; 95 \% \mathrm{Cl} 0.16-0.991)$. Risk factors: a normal relationship with her mother-in-law $(\mathrm{OR}=5.309$; $95 \% \mathrm{Cl} 1.122-4.184)$, artificial insemination $(\mathrm{OR}=4.339$; $95 \% \mathrm{Cl} 1.492-12.623)$, no exercise during pregnancy (OR=2.666; 95\% Cl 1.177-6.039), low self-efficacy $(\mathrm{OR}=4.253 ; 95 \% \mathrm{Cl} 1.518-11.916)$, low social support $(\mathrm{OR}=2.371 ; 95 \% \mathrm{Cl} 1.206-4.661)$, poor sleep quality $(\mathrm{OR}=2.134 ; 95 \% \mathrm{Cl} 1.131-4.027)$, existence of anxiety symptoms ( $\mathrm{OR}=17.654 ; 95 \% \mathrm{Cl} 8.494-36.689)$.

Conclusion The prevalence of depression symptoms is lower than that in developing countries, but due to the large population base of China, the problem should still be taken seriously. To prevent mental disorders of pregnant women, early screening for mental disorders, promotion of healthy lifestyles, mental healthcare during pregnancy and improved family and social support should be implemented during pregnancy nursing.

\section{INTRODUCTION}

Pregnant women undergo role, physiological and psychological changes. Physiological

\section{Strengths and limitations of this study}

This study investigated the depression symptoms of pregnant women after excluding pregnancy complications and explored a wide range of influencing factors, including psychological, physiological and social aspects.

- This study was a larger sample size than other studies in China; the sample was from the community and avoided hospital bias.

- The disadvantage of this study is that the crosssectional design cannot determine the causal relationship between the influencing factors and depressive symptoms.

- The participants' recall may lead to recall bias.

problems may occur during pregnancy, such as constipation, sleepiness, physiological dyspnoea and hormone level fluctuation, while physiological discomfort, hormonal effects and changes in social roles may cause cognitive, behavioural and emotional changes in pregnant women, resulting in mental health problems. These issues are likely to continue to postpartum and can lead to more serious psychological health and fetal adverse outcomes and developmental problems. $^{12}$ The gestation period includes the first trimester (before the 13th weekend), the second trimester (between the 14th and 17 th weekends) and late pregnancy (28 weeks and beyond). Because of the increase of pregnancy duration in late pregnancy, pregnant women's sleep time is shortened, and snoring and the number and times of waking up at night are increased, leading to poor sleep quality and affecting the emotional regulation mechanism of pregnant women. ${ }^{3}$ Studies have shown that women in their third trimester worried more about their baby's 
health and delivery and were at increased risk for depression symptoms. ${ }^{4}$ Therefore, it is of great significance to consider antenatal mental health status and to determine its potential influencing factors by investigating pregnant women in late pregnancy.

There has been a wide range of discussion on prenatal depression. Prenatal depression is a mental disorder during pregnancy characterised by persistent low mood, slow thinking and exercise inhibition. However, the prevalence rates of these conditions were different in different countries based on their measurements and culture background variations. A systematic review performed by retrieving observational studies at three different time periods found that the incidence of depression symptoms in late pregnancy was $12 \% .^{5}$ However, a meta-analysis of prenatal depression in Ethiopia showed that the combined prevalence of prenatal depression was $21.28 \%$ (95\% CI 15.96\%-27.78\%), and the highest prevalence of depressive symptoms was $32.10 \%$ in late pregnancy, with $19.13 \%$ in the first trimester and $18.86 \%$ in the second trimester. ${ }^{6}$

From personal and social perspectives, associated factors for depression in pregnant women mainly included four domains: (1) sociodemographic factors; (2) pregnancy characteristics; (3) personality characteristics and (4) psychosocial factors. For sociodemographic factors such as age, for example, a study on depression symptoms among pregnant women in the middle and third trimesters showed that the younger they are, the higher the positive rate of depression symptoms they have. ${ }^{7}$ Pregnancy characteristics such as planned pregnancy, ${ }^{8}$ history of abortion ${ }^{9}$ and personality traits such as low self-efficacy were related to prenatal depression. ${ }^{10}$ Psychosocial factors such as socioeconomic status, social support and life events were associated with mental health during pregnancy. ${ }^{11}$

Existing research on depression has been more focused on postpartum; reports of depression symptoms in late pregnancy have been less common, and most of them have explored the situation from a single dimension using minor specimens. Therefore, the purpose of the current study was to describe the prevalence of depression symptoms in late pregnancy and to comprehensively analyse its influencing factors. The results of this study may provide scientific basis for the evaluation of mental health status in a prepregnancy examination programme and mental healthcare service to prevent the symptoms of depression in pregnant women.

\section{METHODS}

\section{Design and participants}

This was a cross-sectional survey of pregnant women in late pregnancy in urban areas of Hengyang City, Hunan Province, China, conducted from July to October 2019. The sampling method was a multistage cluster random sampling method. There are five administrative districts in urban Hengyang. In the first stage, one street was randomly selected in each district. In the second stage, because the minimum number of communities on a street is 3,14 communities were randomly selected according to a ratio of $1: 3$, including 4 communities from Zhengxiang street, 3 communities from Qingshan street, 3 communities from Baishazhou street, 2 communities from Guangdong road and 2 communities from Zhurong street.

Inclusion criteria: pregnant women in late pregnancy who had lived in urban areas of Hengyang for more than 6 months and had signed informed consent (gestational week: 28 weeks and beyond). Exclusion criteria: Women with cognitive disorders, severe mental illnesses or other serious diseases and those who could not fill out the questionnaire by themselves.

The sample size was estimated using the sample size calculation formula for cross-sectional studies. According to previous studies, the prevalence of depression symptoms among pregnant women in late pregnancy was $32.1 \%$. ${ }^{6}$ In this study, with admissible error $\mathrm{d}=0.1 \mathrm{p}$, $\mathrm{p}=0.05, \alpha=0.05$, the minimum theoretical sample size for the study was calculated to be approximately 812 people. In fact, 813 pregnant women completed the survey by filling in a paper or electronic WeChat questionnaire after informed consent was obtained, and the results were further analysed.

\section{Measurement \\ Demographic characteristics}

The self-report questionnaire was used to obtain demographic information of pregnant women, including age, ethnicity, marital status, her and her husband's education, occupation, per-capita monthly income and medical expenses payment methods.

\section{Pregnancy characteristics and lifestyle}

Pregnancy characteristics included parity $(0,1, \geq 2)$, methods of pregnancy (artificial insemination/spontaneous pregnancy), whether planned pregnancy, pregnancy complications (including gestational hypertension, gestational diabetes, intrahepatic cholestasis, cervical disease), number of abortions $(0,1, \geq 2)$ and whether regular antenatal examinations were performed.

Lifestyle included prepregnancy body mass index (preBMI), her and her husband's smoking habits, her and her husband's drinking habits and exercise. Pre-BMI was based on the pregnant woman's self-reported height and weight before and after pregnancy using China's classification of thin (BMI $<18.5 \mathrm{~kg} / \mathrm{m}^{2}$ ), normal weight (BMI of $\left.18.5-23.9 \mathrm{~kg} / \mathrm{m}^{2}\right)$ and overweight $\left(\right.$ BMI $>23.9 \mathrm{~kg} / \mathrm{m}^{2}$ ). Smoking was defined as an average of one cigarette a day for the past year. Drinking was defined as drinking alcohol on average once a week. Exercise was defined as walking, yoga or other forms during the past week, yes or no.

\section{Outcome measurements}

Depression symptoms in late pregnancy were assessed using the Patient Health Questionnaire. This questionnaire has been widely used in clinical and observation 
studies with good reliability and validity. There are nine items in this questionnaire, and the score of each item is set to $0-3$, with a total score of 27 . The higher the score, the more obvious the depression symptoms are, with a total score $\geq 10$ considered to be depression symptoms, ${ }^{12} 13$ with a Cronbach's $\alpha$ coefficient of 0.773 .

\section{Family factors}

Family factors include family function, the relationship with the mother-in-law and experience of domestic abuse. Family function was assessed using the Family Adaptation Partnership Growth and Resolve Index (APGAR). The APGAR Scale contains five items and five dimensions, respectively, reflecting the adaptability, cooperation, maturity, affection and affinity of family functions. Each item was scored using a 3-point Likert scale, with a total score of $0-10$, with a score of 7-10 indicating good family function, 4-6 indicating moderate family function disorder and 0-3 indicating severe family function disorder. ${ }^{14}$ APGAR is widely used and has good reliability and validity in pregnant women in China, ${ }^{15}$ with a Cronbach's $\alpha$ coefficient of 0.876 in this study. The Abuse Assessment Screen (AAS) was used to assess domestic violence during pregnancy. Eight items were used to assess exposure to domestic violence in the past year and during pregnancy, including physical, emotional and sexual violence. The respondents answered 'yes' to items $3 / 4 / 5 / 6 / 7$; the total number of times of abuse was more than three times, subjective pain, and the three conditions were considered to have experienced domestic violence. ${ }^{16}$ Research shows that the AAS questionnaire has good reliability and validity in pregnant women, with a Cronbach's $\alpha$ coefficient of 0.685 in this study.

\section{Other relevance factors}

The Social Support Rating Scale ${ }^{17}$ was used in this study to evaluate the status of social support for pregnant women, as introduced by Xiao, and included objective support, subjective support and the availability of support. A higher score means higher social support. A total score less than 39 in this study was considered as low social support. The scale has good reliability and validity, with a Cronbach's $\alpha$ coefficient of 0.705 in this study.

This study used the Pittsburgh Sleep Quality Index (PSQI) to evaluate maternal sleep quality. The total score of PSQI is between 0 and 21 points, and the higher the score, the worse the sleep quality. PSQI Scores $\leq 7$ were rated as 'good sleep quality', while PSQI Scores $>7$ were rated as 'poor sleep quality'. Studies have shown that the PSQI Scale has good reliability and validity in evaluating sleep quality, ${ }^{18}$ and the Chinese version of the PSQI questionnaire has been widely accepted as a sleep quality assessment method in mainland China, with a Cronbach's $\alpha$ coefficient of 0.789 in this study.

The General Self-efficacy Scale (GSES) was used to evaluate pregnant women's self-efficacy. The GSES was developed by German health psychology and clinical experts Zhang and Schwarzer and was translated into Chinese by Zhang. ${ }^{19}$ This scale is a single-dimension scale with 10 items. Each item has four options: completely incorrect, moderately correct, mostly correct and completely correct, marked as 1, 2, 3 and 4, with the total score ranging from 10 to 40 . The total score is divided by the total score of 10 items into low level (0-2), medium level (2.1-3) and high level (3.1-4). The higher the score, the better the self-efficacy is. The Cronbach's $\alpha$ coefficient of this scale was 0.898 in this study.

The Generalised Anxiety Disorder-7 Scale was used in this study to evaluate the anxiety of women in late pregnancy. This table was developed by Spitzer et al and consisted of seven items for screening generalised anxiety disorder. ${ }^{20}$ Participants were asked if they had experienced seven symptoms in the past 2 weeks, with each item being scored on a scale of four, ' $0=$ not at all', ' $1=$ days', ' $2=$ more than half days' and ' $3=$ almost every day', with a score ranging from 0 to 21. Studies have shown that using a cut-off score of 10 is a good way to distinguish anxiety from non-anxiety. ${ }^{21}$ In our study, the Cronbach's $\alpha$ coefficient was 0.773 .

\section{Statistical analysis}

SPSS V.25.0 was used to collate and analyse the data. Continuous variables were converted into categorical variables by referring to the literature and combining research purposes, and all categorical variables are described as counts and percentages. Univariate analysis used the $\chi^{2}$ or Fisher's test to analyse the relationship between sociodemographic characteristics, pregnancy characteristics, lifestyle, family factors, social support, sleep quality, self-efficacy with depressive symptoms and compared the characteristics of between the depression symptoms and non-depression symptoms groups. A multivariate binary logistic regression with ORs was used to analyse the influencing factors of prenatal depression symptoms. Depression symptoms were taken as dependent variables, and factors with statistical significance $(\mathrm{p}<0.05)$ in the univariate analysis were taken as independent variables, including age ( $\leq 24,25-29,30-34, \geq 35)$, pre-BMI (thin, normal, overweight), exercise (yes or no), relationship with mother-in-law (bad, general, good), family function (serious difficulty, moderate difficulty, well), social support (high or low), sleep quality (good or poor) and self-efficacy (low, medium, high). All statistical tests were two-sided.

\section{Patient and public involvement}

Patients and the public were not involved in the design of this study.

\section{RESULTS}

\section{Participants}

Fourteen communities had 819 women in late pregnancy who were registered in community health service centres, but three women in late pregnancy did not conform to the inclusion criteria $(\mathrm{n}=816)$ and three pregnant women 


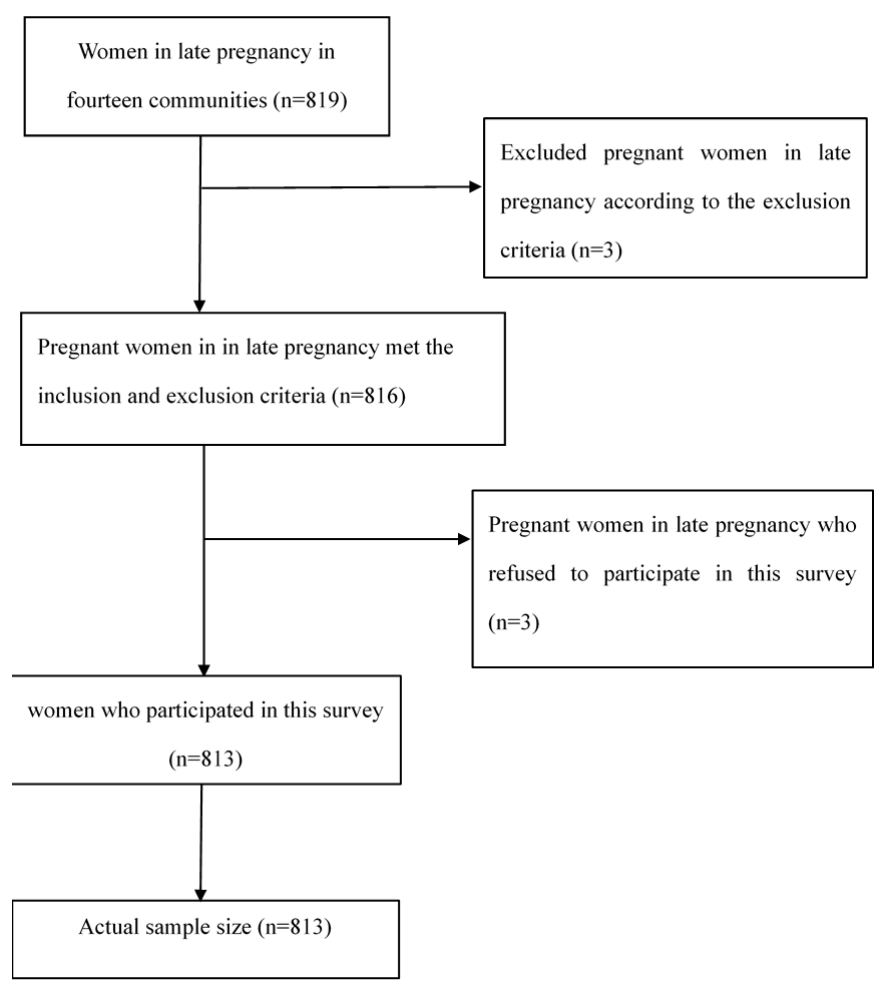

Figure 1 Sample flow chart.

did not complete the questionnaire and refused to participate in the survey $(n=813)$, for a refusal rate of $0.36 \%$. The sampling process is shown in figure 1 .

\section{Sample characteristics}

All participants were aged $17-54$ years (mean=29). Most of them were ethnic Han $(98.2 \%)$. Most were in the state of marriage $(95.4 \%)$. More than half had college/ university and above as their highest education $(58.1 \%)$. Most of the pregnant women were working during pregnancy $(73.7 \%)$. More than half of their husbands had college/university education and above (59.9\%). Most of the husbands were working $(98.3 \%)$. The per-capita monthly income was between $¥ 3001$ and $¥ 7999$ for $70 \%$ of households. The majority of pregnant women $(72.8 \%)$ were covered by medical insurance, as shown in table 1 . A total of $41.6 \%$ were pregnant for the first time. The vast majority $(95.9 \%)$ of pregnant women conceived naturally. More than half $(59 \%)$ were in a planned pregnancy. More than half $(64.0 \%)$ of pregnant women had no history of miscarriage. Most of the pregnant women were without pregnancy complications $(89.5 \%)$. The vast majority of pregnant women received regular prenatal examinations $(91.4 \%)$. Pre-BMI was normal for $67.1 \%$ of pregnant women. A total of $91.4 \%$ of participants exercised during pregnancy, and the results are shown in table 2. More than half $(64.3 \%)$ of the pregnant women had a good relationship with their mother-in-law, and $60.4 \%$ of the pregnant women had good family function. Most of the pregnant women $(90.5 \%)$ did not experience violence during pregnancy. A total of $75.4 \%$ of the pregnant women had good sleep quality. More than half $(54.9 \%)$ of the pregnant women had a high level of social support, and $60.9 \%$ of their self-efficacy was in the medium level; the results are shown in table 3 . In our study, the prevalence rates of depression symptoms and anxiety symptoms were $9.2 \%$ and $7.9 \%$, respectively. The results are shown in tables 1 and 3 .

Influencing factors of depression symptoms in late pregnancy The results of $\chi^{2}$ test showed that age, pre-BMI, relationship with mother-in-law, mode of conception, exercise during pregnancy, sleep quality, self-efficacy, social support and anxiety symptoms were significantly correlated with depression symptoms in late pregnancy (all $\mathrm{p}<0.05$ ). The results are shown in tables $1-3$.

Multivariate binary logistic regression results show that in women, the odds of prenatal depression symptoms were reduced by age between 25 and 29 years $(\mathrm{OR}=0.398$; 95\% CI 0.16-0.991), and the odds of prenatal depression symptoms were increased by artificial insemination during pregnancy $(\mathrm{OR}=4.339 ; 95 \%$ CI $1.492-12.623)$, no exercise during pregnancy $(\mathrm{OR}=2.666$; 95\% CI 1.177-6.039), poor relationships with their mother-in-law $(\mathrm{OR}=5.309$; 95\% CI 1.122-4.184), poor sleep quality $(\mathrm{OR}=2.134$; 95\% CI 1.131-4.027), low self-efficacy (OR=4.253; 95\% CI $1.518-11.916)$, low social support $(\mathrm{OR}=2.371 ; 95 \%$ CI $1.206-4.661)$ and anxiety symptoms $(\mathrm{OR}=17.654 ; 95 \% \mathrm{CI}$ 8.494-36.689). The results are shown in table 4 .

\section{DISCUSSION}

\section{Prevalence of depression symptoms}

The prevalence of depression symptoms in late pregnancy was $9.2 \%$ (95\% CI 7.2\%-11.2\%), indicating lower rates of depression symptoms than those in other developing countries, such as the prevalence rates of depression symptoms reported in Pakistan $(18 \%)^{22}$ and in Thailand $(46.8 \%) .^{23}$ The main reasons for this result are cultural and demographic differences between countries, as well as the adaptability of different scales. ${ }^{24}$ Under the influence of China's 'Saving face culture', people generally have the idea that one should not wash one's dirty linen in public. They are often reluctant to show their weaknesses and bad emotions to outsiders to maintain their own good image and maintain the family's sense of honour and reputation. Thus, pregnant women tend to hide their negative emotions so as not to be seen as weak or discriminated against, which leads researchers to underestimate the positive rates of depression and anxiety symptoms in this group, making our results different from those of other countries.

\section{Influencing factors of depression symptoms in late pregnancy} Demographic factors

Pregnant women between the ages of 25 and 29 had a lower risk of depression than a younger group, in contrast to a meta-analysis in Ethiopia that reported that pregnant women between 20 and 29 were more likely to have symptoms of prenatal depression, ${ }^{6}$ probably because of 
Table 1 Demographic information of pregnant women and distribution of depression symptoms $(n=813)$

\begin{tabular}{|c|c|c|c|c|}
\hline \multirow[b]{2}{*}{ Characteristic } & \multirow[b]{2}{*}{$\begin{array}{l}\text { Total } \\
\text { n (\%) }\end{array}$} & \multicolumn{2}{|c|}{ Depression symptoms } & \multirow[b]{2}{*}{$P$ value } \\
\hline & & $\begin{array}{l}\text { No } \\
\mathrm{n}(\%)\end{array}$ & $\begin{array}{l}\text { Yes } \\
\text { n (\%) }\end{array}$ & \\
\hline $\mathrm{n}$ & $813(100.0)$ & $738(90.8)$ & $75(9.2)$ & \\
\hline Age & & & & 0.017 \\
\hline$\leq 24$ & $115(14.1)$ & $100(87.0)$ & $15(13.0)$ & \\
\hline $25-29$ & $366(45.1)$ & $343(93.7)$ & $23(6.3)$ & \\
\hline $30-34$ & $232(28.5)$ & $202(87.1)$ & $30(12.9)$ & \\
\hline$\geq 35$ & $100(12.3)$ & 93 (93.0) & $7(7.0)$ & \\
\hline Ethnicity & & & & $1.000^{*}$ \\
\hline Minority & $15(1.8)$ & 14 (93.3) & $1(6.7)$ & \\
\hline Han ethnicity & 798 (98.2) & $724(90.7)$ & $74(9.3)$ & \\
\hline Marital status & & & & $0.527^{*}$ \\
\hline Single/divorced/widowed & $37(4.6)$ & $32(86.5)$ & $5(13.5)$ & \\
\hline Married & $776(95.4)$ & $706(91.0)$ & $70(9.0)$ & \\
\hline Education level & & & & 0.577 \\
\hline Junior middle school or below & $158(19.4)$ & $140(88.6)$ & $18(11.4)$ & \\
\hline High school or technical secondary school & $183(22.5)$ & 167 (91.3) & $16(8.7)$ & \\
\hline College or above & $472(58.1)$ & $431(91.3)$ & $41(8.7)$ & \\
\hline Working status & & & & 0.838 \\
\hline Unemployed & $214(26.3)$ & $195(91.1)$ & $19(8.9)$ & \\
\hline Employed & $599(73.7)$ & $543(90.7)$ & $56(9.3)$ & \\
\hline Education level (husband) & & & & 0.704 \\
\hline Junior middle school or below & $122(15.0)$ & $111(91.0)$ & $11(9.0)$ & \\
\hline High school or technical secondary school & $204(25.1)$ & $188(92.2)$ & $16(7.8)$ & \\
\hline College or above & $487(59.9)$ & $439(90.1)$ & $48(9.9)$ & \\
\hline \multicolumn{5}{|l|}{ Working status (husband) } \\
\hline Unemployed & $14(1.7)$ & $14(100.0)$ & $0(0.0)$ & \\
\hline Employed & 799 (98.3) & $724(90.6)$ & $75(9.4)$ & \\
\hline Per-capita monthly income, $¥$ & & & & 0.603 \\
\hline$\leq 3000$ & $73(9.0)$ & $64(87.7)$ & $9(12.3)$ & \\
\hline $3001-7999$ & $572(70.3)$ & $520(90.9)$ & $52(9.1)$ & \\
\hline$\geq 8000$ & $168(20.7)$ & $154(91.7)$ & $14(8.3)$ & \\
\hline Medical expenses payment methods & & & & $0.588^{*}$ \\
\hline Free & $29(3.6)$ & 28 (96.6) & $1(3.4)$ & \\
\hline Out-of-pocket & $192(23.6)$ & $176(91.7)$ & $16(8.3)$ & \\
\hline Medical insurance & $592(72.8)$ & 534 (90.2) & $58(9.8)$ & \\
\hline
\end{tabular}

${ }^{*}$ Continuity correction.

†The signifance of bold font is ' $\mathrm{P}<0.05$ ', and the results are statistically significant.

differences in cultural and educational levels between the two countries. Most pregnant women in this age group already had work experience; thus, they were less worried about finding a job after becoming pregnant. In China, the legal age for marriage and childbirth is 20; thus, they were more likely to experience having children and had more knowledge and skills about pregnancy and childbirth. ${ }^{25}$ In addition, women aged $25-29$ have higher levels of psychological and physical maturity and are better able to withstand stressful life events and prevent depression symptoms.

Pregnancy characteristics and lifestyle factors

Artificial insemination was a risk factor for depression symptoms and has a significant impact on the mental health of pregnant women. The main reason for artificial 
Table 2 Pregnancy characteristics and lifestyle of pregnant women $(n=813)$

\begin{tabular}{|c|c|c|c|c|}
\hline \multirow[b]{2}{*}{ Characteristic } & \multirow[b]{2}{*}{$\begin{array}{l}\text { Total } \\
\text { n (\%) }\end{array}$} & \multicolumn{2}{|c|}{ Depression symptoms } & \multirow[b]{2}{*}{$P$ value } \\
\hline & & $\begin{array}{l}\text { No } \\
\text { n (\%) }\end{array}$ & $\begin{array}{l}\text { Yes } \\
\text { n (\%) }\end{array}$ & \\
\hline Parity & & & & 0.201 \\
\hline 0 & $338(41.6)$ & $314(92.9)$ & $24(7.1)$ & \\
\hline 1 & $413(50.8)$ & $368(89.1)$ & 45 (10.9) & \\
\hline$\geq 2$ & $62(7.6)$ & $56(90.3)$ & $6(9.7)$ & \\
\hline Method of pregnancy & & & & $0.034^{\star}$ \\
\hline Artificial insemination & $33(4.1)$ & $26(78.8)$ & $7(21.2)$ & \\
\hline Spontaneous pregnancy & 780 (95.9) & $712(91.3)$ & $68(8.7)$ & \\
\hline Planned pregnancy & & & & 0.291 \\
\hline Yes & $480(59.0)$ & $440(91.7)$ & $40(8.3)$ & \\
\hline No & $333(41.0)$ & $298(89.5)$ & 35 (10.5) & \\
\hline Number of abortions & & & & 0.112 \\
\hline 0 & $520(64.0)$ & $480(92.3)$ & $40(7.7)$ & \\
\hline 1 & $114(14.0)$ & 99 (86.8) & $15(13.2)$ & \\
\hline$\geq 2$ & $179(22.0)$ & $159(88.8)$ & $20(11.2)$ & \\
\hline Pregnancy complications & & & & 0.646 \\
\hline Yes & $85(10.5)$ & $76(89.4)$ & $9(10.6)$ & \\
\hline No & $728(89.5)$ & $662(90.9)$ & $66(9.1)$ & \\
\hline Regular antenatal examination & & & & 0.126 \\
\hline Yes & $743(91.4)$ & $678(91.3)$ & $65(8.7)$ & \\
\hline No & $70(8.6)$ & $60(85.7)$ & 10 (14.3) & \\
\hline Prepregnancy BMI & & & & 0.002 \\
\hline Thin & $165(20.4)$ & $158(95.8)$ & $7(4.2)$ & \\
\hline Normal & $542(67.1)$ & $479(88.4)$ & $63(11.6)$ & \\
\hline Overweight/obesity & $101(12.5)$ & $97(96.0)$ & $4(4.0)$ & \\
\hline Exercise & & & & 0.001 \\
\hline Yes & $743(91.4)$ & $682(91.8)$ & $61(8.2)$ & \\
\hline No & $70(8.6)$ & $56(50)$ & $14(20.0)$ & \\
\hline
\end{tabular}

${ }^{*}$ Continuity correction.

$\dagger$ The signifance of bold font is ' $P<0.05$ ', and the results are statistically significant.

BMI, body mass index.

insemination was infertility, and women were more likely to feel the stress of infertility. ${ }^{26}$ Because of China's outdated culture of 'having a boy to carry on the family line', artificial insemination, as a manifestation of fertility pressure, continued to affect the mental health of pregnant women. ${ }^{27}$ After successful artificial insemination, the state of pregnancy became the focus of attention of oneself and the family, with worries about loss of the pregnancy, which made pregnant women who were artificially inseminated have more worry and excessive tension than woman with natural pregnancy. ${ }^{28}$

Women who did not exercise during pregnancy might be at greater risk for depression, and exercise during pregnancy could be effective in preventing depression symptoms. Exercise had positive cognitive and emotional benefits while reducing pain and negative effects. ${ }^{29}$
Family factors

As a special culture of developing countries, the relationship culture between mother-in-law and daughter-in-law has been discussed in other articles, and the results were similar to ours. ${ }^{30}{ }^{31}$ In our study, pregnant women with moderate relationships with their mother-in-law were more than twice as likely to have symptoms of prenatal depression as women with good relationships. In Chinese culture, people do not easily report or admit that they have relationship friction or poor relationships with others. Most people were used to using moderate relationships as a substitute, which led to greater risks for pregnant women who have moderate relationships with their mothers-in-law. Due to the one-child policy implemented in China in the 1980s, the relationship between parents and children was an important part of the family 
Table 3 Family factors and other relevance factors of pregnant women $(n=813)$

\begin{tabular}{|c|c|c|c|c|}
\hline \multirow[b]{2}{*}{ Characteristic } & \multirow[b]{2}{*}{$\begin{array}{l}\text { Total } \\
\text { n (\%) }\end{array}$} & \multicolumn{2}{|c|}{ Depression symptoms } & \multirow[b]{2}{*}{$P$ value } \\
\hline & & $\begin{array}{l}\text { No } \\
\text { n (\%) }\end{array}$ & $\begin{array}{l}\text { Yes } \\
\text { n (\%) }\end{array}$ & \\
\hline Relationship with mother-in-law & & & & $<0.001$ \\
\hline $\mathrm{Bad}$ & $27(3.3)$ & $22(81.5)$ & $5(18.5)$ & \\
\hline General & $263(32.3)$ & $226(85.9)$ & $37(14.1)$ & \\
\hline Good & $523(64.3)$ & $490(93.7)$ & $33(6.3)$ & \\
\hline Family function & & & & $<0.001$ \\
\hline Serious difficulty & $66(8.1)$ & $49(74.2)$ & $17(25.8)$ & \\
\hline Moderate difficulty & $256(31.5)$ & $234(91.4)$ & $22(8.6)$ & \\
\hline Well & $491(60.4)$ & $455(92.7)$ & $36(7.3)$ & \\
\hline Domestic violence & & & & 0.107 \\
\hline Yes & $77(9.5)$ & $66(85.7)$ & $11(14.3)$ & \\
\hline No & $736(90.5)$ & $672(91.3)$ & $64(8.7)$ & \\
\hline Sleep quality & & & & $<0.001$ \\
\hline Bad & $200(24.6)$ & $163(81.5)$ & $37(18.5)$ & \\
\hline Good & $613(75.4)$ & $575(93.8)$ & $38(6.2)$ & \\
\hline Self-efficacy & & & & $<0.001$ \\
\hline Low level & $184(22.6)$ & $151(82.1)$ & $33(17.9)$ & \\
\hline Medium level & $495(60.9)$ & $461(93.1)$ & $34(6.9)$ & \\
\hline High level & $134(16.5)$ & $126(94.0)$ & $8(6.0)$ & \\
\hline Social support & & & & $<0.001$ \\
\hline Low & $367(45.1)$ & $313(85.3)$ & $54(14.7)$ & \\
\hline High & $446(54.9)$ & $425(95.3)$ & $21(4.7)$ & \\
\hline Anxiety symptoms & & & & $<0.001$ \\
\hline Yes & $64(7.9)$ & $30(46.9)$ & $34(53.1)$ & \\
\hline No & 749 (92.1) & 708 (94.5) & $41(5.5)$ & \\
\hline
\end{tabular}

The signifance of bold font is ' $P<0.05$ ', and the results are statistically significant.

relationship, and coupled with the influence of family culture and intergenerational transfer, it was common for women to live with their in-laws after marriage in less-developed areas. ${ }^{32}$ Due to the differences in age, culture, education and living habits, the two were prone to conflict since there was no blood ties and because they lived together for a long time. The friction between mother-in-law and daughter-in-law, coupled with the pregnant woman's concern about the conflict, was likely to continue to affect the pregnant woman's mood and produce depression symptoms. ${ }^{31} 33$

\section{Other factors}

Perinatal depression symptoms were associated with low self-efficacy in pregnant women, which was related to the mediating effect of self-efficacy on social support, pregnancy stress and depression symptoms. ${ }^{34}$ Pregnant women with high levels of self-efficacy could actively face stressful events in life, which was more advantageous for enhancing self-efficacy and thus effectively regulating mood and the prevention of depression symptoms.
Consistent with other findings, poor sleep quality was associated with depression symptoms in late pregnancy. ${ }^{35}$ Due to night urine, fetal movement, leg cramps and other symptoms, the number and time pregnant women awakened during the night would increase, and the sleep time shortened, resulting in poor sleep quality; thus, depression symptoms were related to sleep disorders in late pregnancy. ${ }^{36}$

Lack of social support was a risk factor for depression symptoms; pregnant women could direct the effects on depression of pregnant women. ${ }^{37}$ As an important social resource for pregnant women, social support function was a social determinant of mental health. Positive social support made people feel concerned, loved and valued, which could improve the quality of life of pregnant women and promote their mental health. ${ }^{38} 39$

Consistent with the results of previous studies, pregnant women with anxiety during pregnancy were more likely to suffer from depression symptoms. ${ }^{11}{ }^{40}$ Depression and anxiety symptoms have similarities; therefore, they are 
Table 4 Factors associated with depression symptoms in late pregnancy $(n=813)$

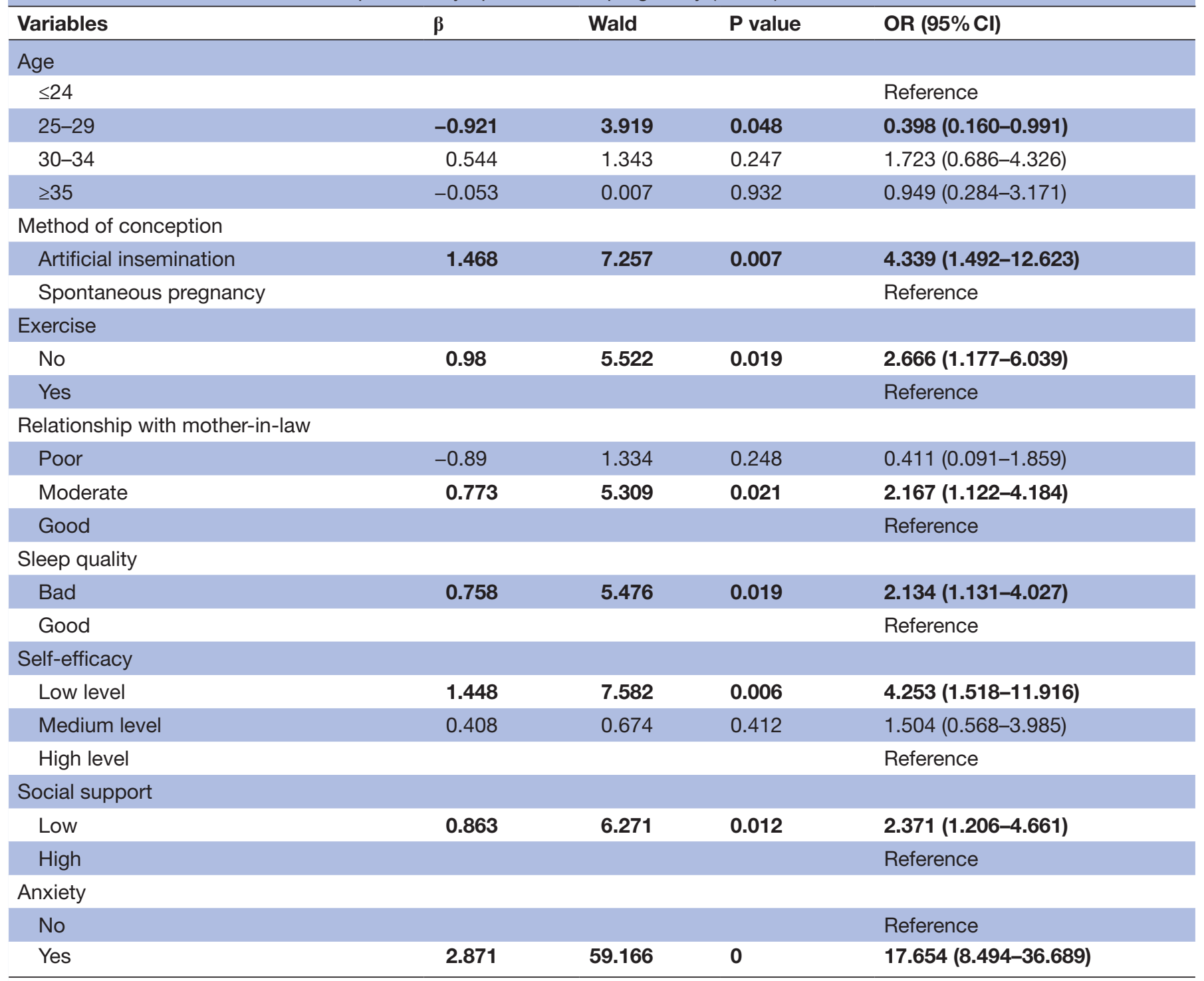

The bold font indicates that the results are statistically significant.

often found to coexist. ${ }^{41}$ In addition, different emotional problems would affect each other, increasing the risk of other emotions. ${ }^{11}$

\section{LIMITATION}

The limitations of this study were that cross-sectional data were used to determine the causal relationship between risk factors and depression, and there were options in the questionnaire that needed to be filled out by participants recalling the situations of the last week, month or year, meaning that there was recall bias. However, this questionnaire selected a measurement tool with certain reliability and validity to minimise this problem. Additionally, the data were from women in late pregnancy in an urban area of Hengyang city; thus, the results could not be extended to rural areas. However, due to the urbanisation process and intergenerational transfer process in
China, this study had greater predictive significance. In addition, after screening pregnant women for depression symptoms, no further diagnosis and intervention was performed.

\section{CONCLUSION}

Our study reported that the prevalence rate of depression symptoms was $9.2 \%$ in pregnant women in Hengyang city. The influencing factors were age, relationship with the mother-in-law, mode of pregnancy, exercise during pregnancy, sleep quality, self-efficacy, social support and symptoms of anxiety symptoms. These findings suggested that the medical institutions should add mental health screening tools in pregnancy examinations to realise early discovery, early diagnosis and early treatment. When implementing maternal healthcare in medical institutions and community health service institutions, we should pay 
attention to the psychological status of pregnant women and provide them with targeted maternal health nursing.

Acknowledgements The author would like to thank all members of the research team for their hard work in the process of research design and data collection and for the academic guidance and assistance of team members in the process of paper writing.

Contributors YY, XZ and ZH contributed to conceptualisation, methodology and software; $Y Y$ contributed to data curation, writing, original draft preparation and reviewing; $\mathrm{YY}, \mathrm{XZ}, \mathrm{ZH}, \mathrm{WZ}, \mathrm{BZ}$ and SY contributed to visualisation and investigation; $H X$ contributed to supervision.

Funding The authors have not declared a specific grant for this research from any funding agency in the public, commercial or not-for-profit sectors.

Disclaimer The views expressed in this article belong to the authors and are not to the official position of any other institution or funder.

Competing interests None declared.

Patient and public involvement Patients and/or the public were not involved in the design, or conduct, or reporting, or dissemination plans of this research.

Patient consent for publication Not required.

Ethics approval All respondents gave written informed consent before entering the study and volunteered for the survey. The study was approved by the Ethics Committee of the Xiangya School of Public Health, Central South University (XYGW2019-056), prior to the field investigation.

Provenance and peer review Not commissioned; externally peer reviewed.

Data availability statement Data sharing not applicable as no datasets generated and/or analysed for this study. No additional data available.

Open access This is an open access article distributed in accordance with the Creative Commons Attribution Non Commercial (CC BY-NC 4.0) license, which permits others to distribute, remix, adapt, build upon this work non-commercially, and license their derivative works on different terms, provided the original work is properly cited, appropriate credit is given, any changes made indicated, and the use is non-commercial. See: http://creativecommons.org/licenses/by-nc/4.0/.

\section{ORCID iDs}

Huilan Xu http://orcid.org/0000-0002-2202-8675

Zhao Hu http://orcid.org/0000-0002-6787-4074

Wensu Zhou http://orcid.org/0000-0002-3871-1479

\section{REFERENCES}

1 Walder DJ, Laplante DP, Sousa-Pires A, et al. Prenatal maternal stress predicts autism traits in $61 / 2$ year-old children: project ice storm. Psychiatry Res 2014;219:353-60.

2 Staneva A, Bogossian F, Pritchard M, et al. The effects of maternal depression, anxiety, and perceived stress during pregnancy on preterm birth: a systematic review. Women Birth 2015;28:179-93.

3 Bayrampour H, McDonald S, Tough S. Risk factors of transient and persistent anxiety during pregnancy. Midwifery 2015;31:582-9.

4 Bisetegn TA, Mihretie G, Muche T. Prevalence and predictors of depression among pregnant women in Debretabor town, Northwest Ethiopia. PLoS One 2016;11:e0161108.

5 Bennett HA, Einarson A, Taddio A, et al. Prevalence of depression during pregnancy: systematic review. Obstet Gynecol 2004;103:698-709.

6 Ayano G, Tesfaw G, Shumet S. Prevalence and determinants of antenatal depression in Ethiopia: a systematic review and metaanalysis. PLoS One 2019;14:e0211764.

7 Bowen A, Bowen R, Maslany G, et al. Anxiety in a socially highrisk sample of pregnant women in Canada. Can J Psychiatry 2008:53:435-40.

8 Mohammad KI, Gamble J, Creedy DK. Prevalence and factors associated with the development of antenatal and postnatal depression among Jordanian women. Midwifery 2011;27:e238-45.

9 Scheidt CE, Kunze M, Wangler J, et al. Psychological consequences of perinatal loss in subsequent pregnancies-a comparative study]. Psychother Psychosom Med Psychol 2008;58:475-8.

10 Kossakowska K. Incidence and determinants of postpartum depression among healthy pregnant women and high-risk pregnant women. Postępy Psychiatrii i Neurologii 2016;25:1-21.
11 Tang X, Lu Z, Hu D, et al. Influencing factors for prenatal stress, anxiety and depression in early pregnancy among women in Chongqing, China. J Affect Disord 2019;253:292-302.

12 Kroenke K, Spitzer RL, Williams JB. The PHQ-9: validity of a brief depression severity measure. J Gen Intern Med 2001;16:606-13.

13 Chin W-Y, Wan EYF, Dowrick C, et al. Tree analysis modeling of the associations between PHQ-9 depressive symptoms and doctor diagnosis of depression in primary care. Psychol Med 2019:49:449-57.

14 Smilkstein G, Ashworth C, Dan M. Validity and reliability of the family Apgar as a test of family function. J Fam Pract 1982;15:303-11.

15 Liu Y, Xiu-ling D, Li Z. Influenceofsocialsupportandfamily functionontheapproachofdelivery. Chinese mordern clinical medicine 2012;38:358-61.

16 Reichenheim ME, Moraes CL. Comparison between the abuse assessment screen and the revised conflict tactics scales for measuring physical violence during pregnancy. $J$ Epidemiol Community Health 2004;58:523-7.

17 Xiao SY. The social support rating scale. Chin Ment Health J 1999;12:127-31.

18 Liu TM. Reliabilty and validaity of the Pittsburgh sleep quality index. Chinese Journal of Psychiatry 1996;29:103-7.

19 Zhang JX, Schwarzer R. Measuring optimistic self-beliefs: a Chinese adaptation of the general self-efficacy scale. Psychologia An International Journal of Psychology in the Orient 1995;38:174-81.

20 Spitzer RL, Kroenke K, Williams JBW, et al. A brief measure for assessing generalized anxiety disorder. Arch Intern Med 2006;166:1092-7.

21 Schalet BD, Cook KF, Choi SW, et al. Establishing a common metric for self-reported anxiety: linking the MASQ, PANAS, and GAD-7 to PROMIS anxiety. J Anxiety Disord 2014;28:88-96.

22 Karmaliani R, Asad N, Bann CM, et al. Prevalence of anxiety, depression and associated factors among pregnant women of Hyderabad, Pakistan. Int J Soc Psychiatry 2009;55:414-24.

23 Phoosuwan N, Eriksson L, Lundberg PC. Antenatal depressive symptoms during late pregnancy among women in a north-eastern Province of Thailand: prevalence and associated factors. Asian $J$ Psychiatr 2018;36:102-7.

24 Halbreich U, Karkun S. Cross-Cultural and social diversity of prevalence of postpartum depression and depressive symptoms. $J$ Affect Disord 2006;91:97-111.

25 Qiao Y-X, Wang J, Li J, et al. The prevalence and related risk factors of anxiety and depression symptoms among Chinese pregnant women in Shanghai. Aust N Z J Obstet Gynaecol 2009;49:185-90.

26 Hosaka T, Matsubayashi H, Sugiyama Y, et al. Effect of psychiatric group intervention on natural-killer cell activity and pregnancy rate. Gen Hosp Psychiatry 2002;24:353-6.

$27 \mathrm{~m} \mathrm{H}$, Ren J, Gao P. A related study of depression and anxiety in female patients with infertility. Chinese psychiatry 2017;30:438-40.

28 Ren LJ, Zhang Y, Xu Q. Analysis of anxiety and depression in pregnant women at different stages of pregnancy and its influencing factors. Chinese people's military surgeon 2018;61:797-801.

29 Lindwall M, Josefsson T, Archer T. Effects of physical exercise on depressive symptoms and biomarkers in depression. Cns Neurol Disord Drug Targets 2014;13:1640-53.

30 Fisher J, Tran T, La BT, et al. Common perinatal mental disorders in northern Viet Nam: community prevalence and health care use. Bull World Health Organ 2010;88:737-45.

31 Wan EY, Moyer CA, Harlow SD, et al. Postpartum depression and traditional postpartum care in China: role of Zuoyuezi. Int $J$ of Obstet 2009;104:209-13.

32 Jiang Q, Liu Y. Low fertility and concurrent birth control policy in China. Hist Fam 2016;21:551-77.

33 Fisher J, Cabral de Mello M, Patel V, et al. Prevalence and determinants of common perinatal mental disorders in women in low- and lower-middle-income countries: a systematic review. Bull World Health Organ 2012;90:139-49.

34 Razurel C, Kaiser B, Antonietti J-P, et al. Relationship between perceived perinatal stress and depressive symptoms, anxiety, and parental self-efficacy in primiparous mothers and the role of social support. Women Health 2017;57:154-72.

35 Polo-Kantola P, Aukia L, Karlsson $\mathrm{H}$, et al. Sleep quality during pregnancy: associations with depressive and anxiety symptoms. Acta Obstet Gynecol Scand 2017;96:198-206.

36 Mindell JA, Cook RA, Nikolovski J. Sleep patterns and sleep disturbances across pregnancy. Sleep Med 2015;16:483-8.

37 Lancaster CA, Gold KJ, Flynn HA, et al. Risk factors for depressive symptoms during pregnancy: a systematic review. Am J Obstet Gynecol 2010;202:5-14. 
38 Giesbrecht GF, Poole JC, Letourneau N, et al. The buffering effect of social support on hypothalamic-pituitary-adrenal axis function during pregnancy. Psychosom Med 2013;75:856-62.

39 Thompson T, Perez M, Kreuter M, et al. Perceived social support in African American breast cancer patients: predictors and effects (vol 192, PG 134, 2017). Soc Sci Med 2019;223:117-20.
40 Grant K-A, McMahon C, Austin M-P. Maternal anxiety during the transition to parenthood: a prospective study. J Affect Disord 2008;108:101-11.

41 Clark LA, Watson D. Tripartite model of anxiety and depression: psychometric evidence and taxonomic implications. J Abnorm Psychol 1991;100:316-36. 